\title{
Performance of Time Domain Migration Influenced by Non-Ideal UWB Antennas
}

\author{
Malgorzata Janson, Student Member, IEEE, Thomas Zwick, Senior Member, IEEE, and \\ Werner Wiesbeck, Fellow, IEEE
}

\begin{abstract}
The antennas in ultrawideband (UWB) systems act as filters in both frequency domain and the spatial domain. This means that the antenna influence on a received signal varies with the wave incidence angle. This is particulary important for imaging systems, which make use of the time information of the received signals. However, since the incidence angles of the signals are not a priori known it is difficult to remove this effect from the measured data. To asses the antenna impact on the performance of the imaging system, in this work, simulated scattered signals are used as input for time domain migration algorithm. Then the image features like position errors, shape of the target image, resolution and coverage of an imaging system are extracted and compared for different real UWB antennas.
\end{abstract}

Index Terms-Time domain migration, ultrawideband (UWB) antennas, ultrawideband imaging.

\section{INTRODUCTION}

$\mathbf{T}$ HE first ultrawideband (UWB) transmission experiments by Heinrich Hertz in Karlsruhe in 1887 used a spark-gap transmitter, which naturally generates impulse signals with a very wide bandwidth. Later on narrowband systems gained the superiority. Until recently, the UWB systems have been primarily used in military applications like radar or secure communications. Due to the approval of unlicensed operation of UWB systems by the Federal Communications Commission (FCC) in 2002 [1] and by the European Commission in 2007 [2] the way for commercial UWB applications has been opened.

While high-rate communication systems seem to be the main application of UWB, also localization and radar systems can profit from the extremely large bandwidth [3], [4]. In recent years a number of approaches for UWB imaging and target identification have been successfully tested [5]-[9]. These approaches, utilizing the information in the time delay, are sensitive to pulse distortions and additional delays generated in the antennas. The influence of such distortions on the target position, image resolution and coverage has been shown in [10]. In this paper additionally the shape of the target image is considered as well as the influence of the antenna radiation center shift with respect to the origin of the coordinate system used for imaging. Also influences of some other system parameters like

Manuscript received October 22, 2008; revised April 02, 2009. First published September 04, 2009; current version published November 04, 2009. This work was supported by the Deutsche Forschungsgesellschaft (DFG) within the priority programme 1202 "UWB Radio Technologies for Communication, Localization and Sensor Technology (UKoLos)."

The authors are with Institut für Hochfrequenztechnik und Elektronik, Universität Karlsruhe (TH), Kaiserstr 12, 76131 Karlsruhe, Germany (e-mail: malgorzata.janson@ihe.uka.de; thomas.zwick@ihe.uka.de; werner.wiesbeck@ihe.uka.de).

Digital Object Identifier 10.1109/TAP.2009.2031664

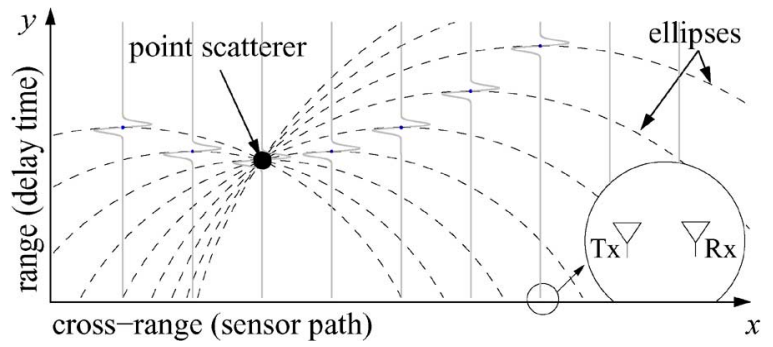

Fig. 1. Time domain migration principle.

bandwidth, aperture and the antenna spacing on the image are discussed for sake of completeness.

The remainder of this paper is organized as follows: First the description of the imaging approach is given, followed by the introduction of the scenario and of the simulation method. Next, the considered UWB antennas are presented. Finally the achieved results are described and the conclusions presented.

\section{IMAGING APPROACH}

The considered imaging method is the time domain migration [5], [6], [9]. A sensor, consisting of the transmit (Tx) and receive (Rx) antennas, is moved in steps along a linear path. At each position the scattered signal is recorded. Assuming that an ideal point scatterer is present, the transmitted pulse will be delayed by the time corresponding to the forward and return path of the signal. With changing position of the sensor the delay of the received pulse changes, resulting in a hyperbolic wave front in the collected data.

The information about the delay at each particular sensor position allows to construct an ellipse with the foci at the positions of Tx and Rx. The length of the semi-major axis corresponds to the delay of the signal. In the monostatic case, when the Tx and $\mathrm{Rx}$ are co-positioned, the ellipse is degenerated to a circle. This ellipse corresponds to all possible scatterer positions. The ellipses for all sensor positions have one common point at the real position of the scatterer (cf. Fig. 1).

An image of the whole considered area can be obtained in the following way: first a grid for the image $O(x, y)$ is generated for the area under consideration. For each point of the grid a point scatterer is assumed, and the distance between this scatterer and the sensor is calculated, then the values of the measured data are found for this distance. Repeating this procedure for all antenna positions delivers the final image. This is expressed by the following:

$$
O(x, y)=\sum_{n=1}^{N} h_{n}\left(\frac{r_{T x_{n}}+r_{R x_{n}}}{c_{0}}\right)
$$




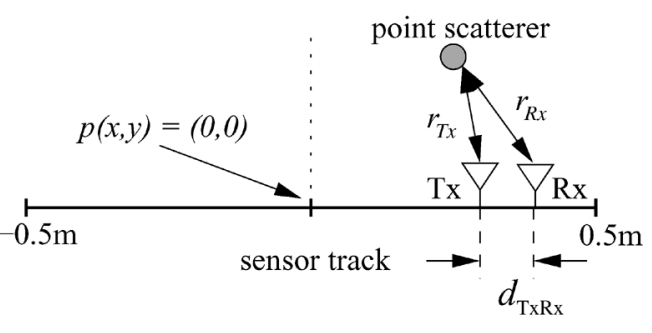

Fig. 2. Imaging scenario.

where $h_{n}(t)$ is the impulse response measured at the $\mathrm{n}$-th receiver position. $r_{T x_{n}}$ is the distance between the transmitter and the considered scatterer and $r_{R x_{n}}$ is the distance between the scatterer and the receiver, and $c_{0}$ indicates the velocity of light.

\section{SCENARIO AND SimUlation APPROACH}

The considered test scenario consists of the sensor and single or multiple point scatterers and is shown in Fig. 2. In the following a $l=1 \mathrm{~m}$ long track with 51 sensor positions at $2 \mathrm{~cm}$ spacing is considered. The Tx and Rx antennas are placed at the same height, the spacing between them is $d_{T x R x}=15 \mathrm{~cm}$ in the $x$-direction. These are the parameter of a measurement system, which was used for experimental analysis of sample scenarios. Some corresponding results for a different track length and different spacing between the Tx and Rx antennas have been presented in [10]. The positions of the target are given in respect with the point $p=(0 \mathrm{~m}, 0 \mathrm{~m})$, which corresponds to the center of the sensor track.

The received signal in such a scenario is influenced by the scattering properties of the target, by the distances of the target to the transmitter $r_{\mathrm{Tx}_{\mathrm{n}}}$ and to the receiver $r_{\mathrm{Rx}_{\mathrm{n}}}$ and by the antennas. In the frequency domain the wave propagation can be described by (2) [11], [12], where $\underline{H}(f)$ is the complex transfer function of the channel, including the antennas. $G$ describes the gain of the antenna and $\underline{C}_{\theta}$ and $\underline{C}_{\psi}$ describe the complex pattern of the transmit and the receive antennas. The indices $\theta$ and $\psi$ relate to the spherical coordinate system shown in Fig. 3

$$
\begin{aligned}
& \underline{H}(f)=\sqrt{\frac{\lambda_{0}^{2} G_{\mathrm{Rx}}(f) G_{\mathrm{Tx}}(f)}{4 \pi}} \\
& \times\left(\left[\underline{C}_{\theta, \mathrm{Rx}}(\theta, \psi, f) \underline{\underline{C}}_{\psi, \mathrm{Rx}}(\theta, \psi, f)\right]^{T} \frac{e^{-j k_{0} r}}{r}\left[\begin{array}{cc}
1 & 0 \\
0 & -1
\end{array}\right]\right. \\
& \left.\times\left[\begin{array}{l}
\underline{C}_{\theta, \mathrm{Tx}}(\theta, \psi, f) \\
\underline{\underline{C}}_{\psi, \mathrm{Tx}}(\theta, \psi, f)
\end{array}\right]\right) \\
& +\sqrt{\frac{\lambda_{0}^{2} G_{R x}(f) G_{T x}(f)}{4 \pi}} \\
& \times\left(\left[\underline{C}_{\theta, \mathrm{Rx}}(\theta, \psi, f) \underline{\underline{C}}_{\psi, \mathrm{Rx}}(\theta, \psi, f)\right]^{T} \frac{e^{-j k_{0} r_{\mathrm{Rx}}}}{r_{\mathrm{Rx}}}\right. \\
& \times\left[\begin{array}{ll}
S_{\theta, \theta}(\theta, \psi, f) & S_{\theta, \psi}(\theta, \psi, f) \\
S_{\psi, \theta}(\theta, \psi, f) & S_{\psi, \psi}(\theta, \psi, f)
\end{array}\right] \frac{e^{-j k_{0} r_{\mathrm{Tx}}}}{r_{\mathrm{Tx}}} \\
& \left.\times\left[\begin{array}{l}
\underline{C}_{\theta, \mathrm{Tx}}(\theta, \psi, f) \\
\underline{\underline{C}}_{\psi, \mathrm{Tx}}(\theta, \psi, f)
\end{array}\left[\begin{array}{l}
\underline{C}_{\theta, \mathrm{Rx}}(\theta, \psi, f) \\
\underline{\underline{C}}_{\psi, \mathrm{Rx}}(\theta, \psi, f)
\end{array}\right]^{T}\right]\right)
\end{aligned}
$$

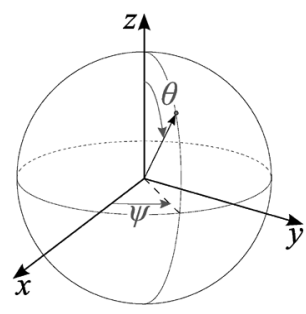

Fig. 3. Coordinate system.

The first term in the equation corresponds to the free space coupling between both antennas. This term is undesirable for any kind of imaging system and is usually removed from the received signal by the system calibration or gating in the time domain. The second term describes the free space propagation between the transmitter and the scatterer, the scattering process and the free space propagation between the scatterer and the receiver. The coefficients $S_{p, q}$ in the scattering matrix depend on frequency, polarization and the directions of the incident and scattered paths. The indices $p$ and $q$ indicate the polarization of the scattered and of the incident waves. Commonly, the scattering of an object is described in terms of a radar cross section (RCS) matrix $\left[\sigma_{\mathrm{p}, \mathrm{q}}\right]$, where $\left[\sigma_{\mathrm{p}, \mathrm{q}}\right]=4 \pi\left|S_{\mathrm{p}, \mathrm{q}}^{2}\right|[11]$.

As this work concentrates on the influence of antennas on the image, the scatterer is assumed to be ideal. Thus the scattering matrix of the target is defined as

$$
S_{p, q}^{\mathrm{ref}}=\left[\begin{array}{cc}
1 & 0 \\
0 & -1
\end{array}\right] \text {. }
$$

All scattering coefficients are assumed to be independent on frequency and incident/scattered angles. Multiple wave interactions with the objects (multipath components) are neglected in the following. Their influence is described in [13].

For each sensor position the distances to the scatterer are calculated and the incidence/scattering angles are determined. The free space propagation terms are multiplied with the antenna pattern components $\underline{C}_{\theta, \phi}$, evaluated for the direction of departure at the transmitter and for the direction of the arrival at the receiver. In the following only vertical polarization of the transmit and receive antennas is considered, but the horizontal polarization can be calculated in the same way. Repeating this process for a range of frequencies in the UWB band, the transfer function of the channel is determined and transformed into time domain by Fourier transform. In this way an ideal propagation scenario with real antennas is obtained. The following results have been obtained in the FCC UWB frequency range from $3.1 \mathrm{GHz}$ to $10.6 \mathrm{GHz}$.

Also other frontend analog components like amplifiers or filters and cables can be considered in the simulation using the following:

$$
\underline{H}_{\text {sys }}(f)=\underline{H}_{\text {frontend,Rx }}(f) \cdot \underline{H}(f) \cdot \underline{H}_{\text {frontend,Tx }}(f) .
$$

As the migration processing takes place in the digital part of the system, it is necessary to include all elements of the system between the channel and the A/D converter. The transfer function of the system $\underline{H}_{\text {sys }}(f)$ can be calculated in the frequency domain by the multiplication of transfer functions of the single 


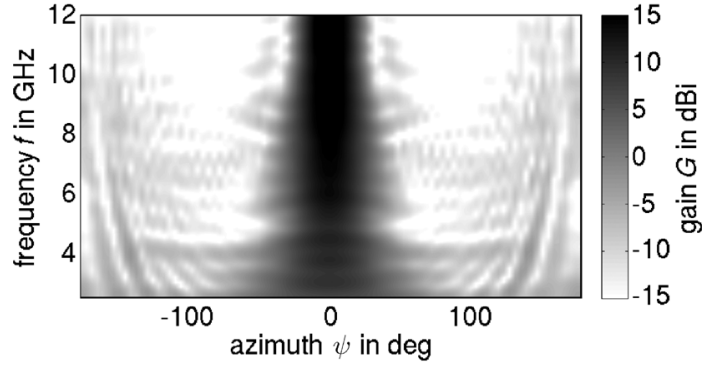

(a)

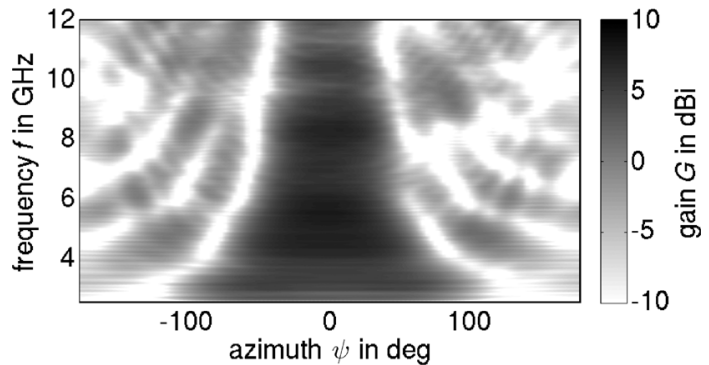

(b)

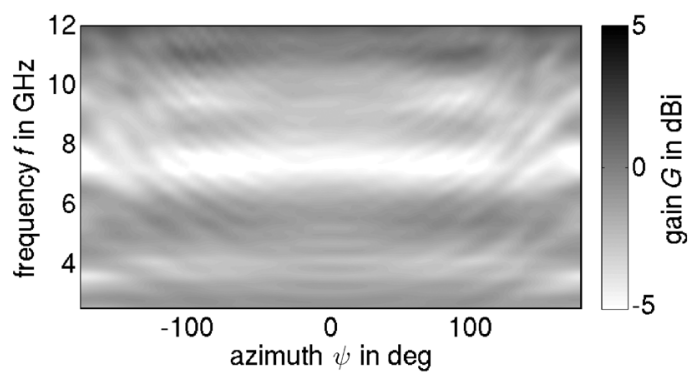

(c)

Fig. 4. Measured gain versus the frequency. (a) Quad ridged horn antenna; (b) Vivaldi antenna; (c) monocone antenna.

frontend elements (4). These elements, however, do not change their behavior as a function of angle $(\theta, \psi)$ like the antennas. Thus their characteristics can be easily deconvolved from the received signal.

\section{ANTENNAS FOR UWB}

The ultrawideband systems have specific requirements on the antennas. Not only the radiation pattern and the gain are important for the performance of a system, also their frequency dependence and thus their time domain behavior has to be considered. For impulse radio UWB systems antennas with a flat transfer function and constant group delay are necessary, otherwise the transmitted pulse will be distorted. Moreover the transfer function of the antenna changes with the observation angle. This results in different delay times in the time domain. As the considered imaging approach utilizes the information about the propagation time, this additional delay can lead to uncertainties in the image. This effect, however, cannot be easily calibrated in the localization or imaging systems as the angles of departure or arrival are not a priori known.

To analyze the impact of this effect on the imaging algorithm, different antennas are inserted into the simulation described in
TABLE I

CHARACTERISTICS OF THE ANTENNAS FOR $f_{\min }=3.1 \mathrm{GHz}$ AND $f_{\max }=10.6 \mathrm{GHz}$

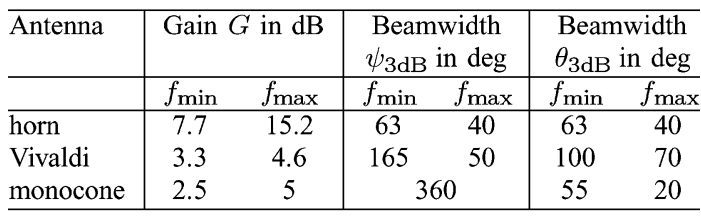

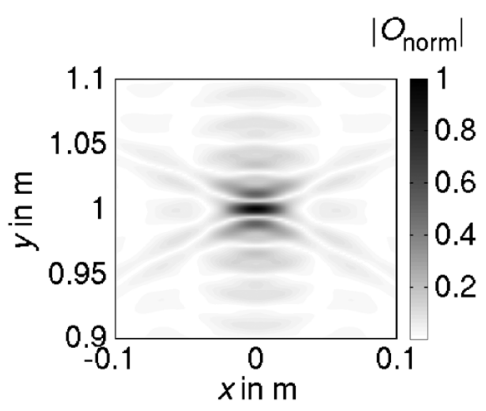

Fig. 5. Normalized image $O(x, y)$ of an ideal scatterer, obtained with ideal, isotropic frequency independent antenna, $p(x, y)=(0 \mathrm{~m}, 1 \mathrm{~m})$

Section III. All these antennas have good time domain parameters [14] but different patterns and different angular dependent behavior of the transfer functions.

As a reference a fictive, ideal isotropic antenna is used. This antenna has a constant transfer function versus frequency and angle and thus it does not distort the pulse in any way. Three real UWB antennas are considered: two directive antennas including a commercially available broadband quad ridged horn antenna (EM Systems, A6100), a Vivaldi antenna [14], and an omnidirectional monocone antenna. The characteristics of these antennas have been measured in an anechoic chamber for the FCC UWB frequency range. In Fig. 4 the resulting gain versus the frequency and the angle in the azimuth plane ( $\psi$ in Fig. 3$)$ is depicted. The azimuth $\psi$ corresponds to the H-plane and elevation $\theta$ to the E-plane of the antennas. The main beam characteristics of the considered antennas are summarized in Table I.

The monocone antenna shows the least angle dependence of the gain and the transfer function in the azimuth plane, whereas the horn antenna the largest one. Already at the angle of $\psi=$ $45^{\circ}$ the gain values are $10 \mathrm{~dB}$ (at $\left.3 \mathrm{GHz}\right)$ to $30 \mathrm{~dB}$ (at $10 \mathrm{GHz}$ ) below the values at the angle of $\psi=0^{\circ}$.

\section{INFLUENCE OF ANTENNAS}

\section{A. Image Characteristics}

In an ideal case (ideal omnidirectional antennas, no frequency dependency of the gain) the migration contributions from all sensor positions cross exactly in one point as shown in Fig. 1. In Fig. 5 an image of a scatterer at the position $p(x, y)=(0 \mathrm{~m}, 1 \mathrm{~m})$ is depicted. The grayscale values in the image correspond to the absolute value of $O(x, y)$, normalized by the maximum value in the image. The image results from simulated data for a $l=1 \mathrm{~m}$ long track with 51 sensor positions. The Tx and Rx antennas are placed at the same height, the spacing between them is $15 \mathrm{~cm}$ in the $x$-direction. It is 


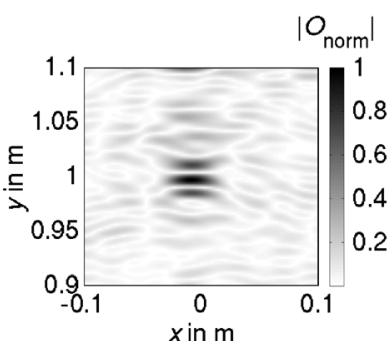

(a)

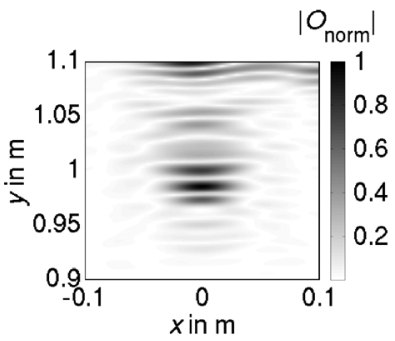

(c)

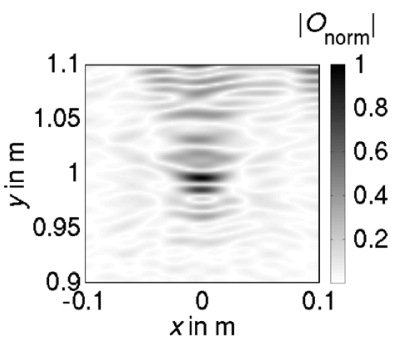

(e)

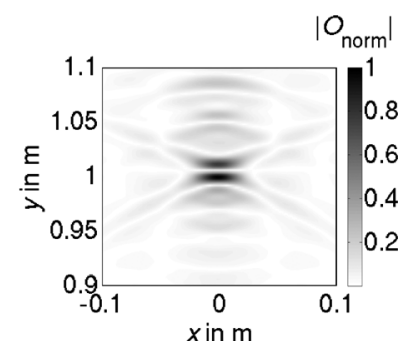

(b)

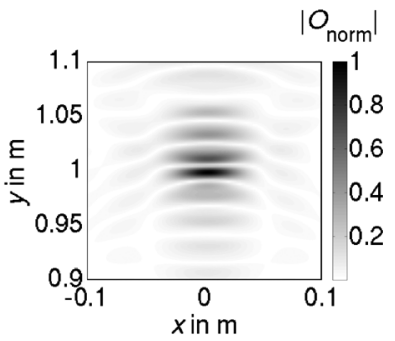

(d)

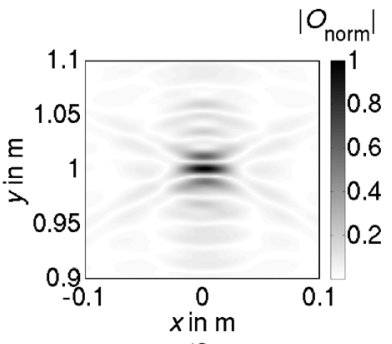

(f)
Fig. 6. Image of a single point target, $p(x, y)=(0 \mathrm{~m}, 1 \mathrm{~m})$. (a) Monoconemeasurement; (b) monocone-simulation; (c) horn-measurement; (d) horn-simulation; (e) Vivaldi-measurement; (f) Vivaldi-simulation.

clearly visible that the image is exactly centered at the real position of the scatterer.

In real UWB antennas effects like creeping waves, energy storage and shifting of the phase center with frequency cause distortions and delays of the radiated pulse. For example in the case of the horn and Vivaldi antennas, which use the travelling wave principle, the antenna structure delays the pulses at angles outside of the main beam. This causes the variance of the transient antenna response and transfer function over the observation angle and the distortion of the image of the target.

Also the directivity of the antenna has an influence on the image shape. Whereas the omnidirectional antennas can capture the signal scattered at the target from all positions along the track, directive antennas attenuate the signals coming from outside of the main beam considerably. The sensor positions with high incidence angles have only a limited contribution to the image. Thus the effective length of the track becomes shorter and the size of the target image in the cross-range becomes wider.

In real measurement systems the frequency dependence of the antennas as well as the influences of other frontend elements like amplifiers, filters etc. are calibrated from the measured data using the main beam through calibration: the transmitter and receiver are placed face to face at some particular distance so that the line of sight conditions are fulfilled and no multipath occurs. The obtained signal can be mathematically described as a

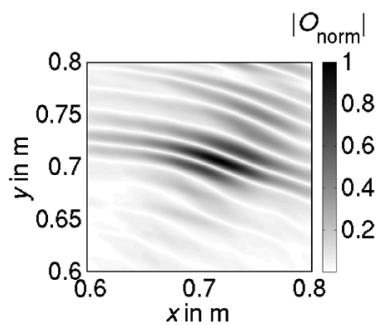

(a)

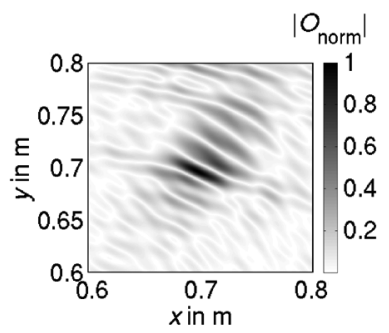

(c)

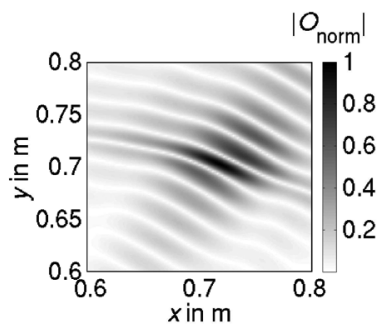

(b)

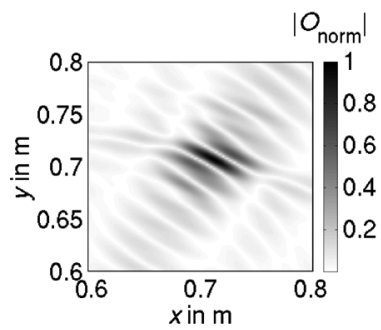

(d)
Fig. 7. Image of a single point target, $p(x, y)=(0.71 \mathrm{~m}, 0.71 \mathrm{~m})$. (a) Horn-measurement; (b) horn-simulation; (c) Vivaldi-measurement; (d) Vivaldi-simulation.

convolution of impulse responses of the channel, frontend elements, cables, and antennas in the direction of $\psi=0^{\circ}, \theta=90^{\circ}$. For the free space channel the hardware transfer function can be easily extracted and deconvolved from the measured data.

Fig. 6 depicts the measured and simulated images of a point target at position $p(x, y)=(0 \mathrm{~m}, 1 \mathrm{~m})$ for all antennas. The images in Fig. 6(a), (c), and (e) have been obtained from measured data. For the measurements a network analyzer is used. The frequency range is $3.1-10.6 \mathrm{GHz}$. A metallic sphere with a diameter of $d=5 \mathrm{~cm}$ is the target. The images in Fig. 6(b), (d), and (f) are simulation results obtained with the method described in Section III. In both cases the impulse response of the antenna in the main beam direction is deconvolved from the scattered signals. This removes the effects of the frequency dependent antenna gain for the targets in the front of the antennas.

The simulated images are almost identical to the measured ones, although in the latter the target position in the image is shifted slightly in some cases. This is due to uncertainties of the target placement during the measurement. As expected, the image obtained with the horn antenna is wider than the other images because of its high directivity.

If the scattered wave arrives at the receiver at an angle different than $\theta=90^{\circ}, \psi=0^{\circ}$ with respect to the center of the sensor path, the calibration does not remove all the influences of the antenna. In Fig. 7 a point scatterer is placed at the position $p(x, y)=(0.71 \mathrm{~m}, 0.71 \mathrm{~m})$ - this corresponds to the distance $d=1 \mathrm{~m}$ and an angle of $\alpha=45^{\circ}$ with respect to the center of the sensor track.

Both images are less focused than for the direction of $0^{\circ}$, however, for the Vivaldi antenna the image is placed at the correct position. The horn antenna is more sensitive to the influence of the angle dependent transfer function-the position of the image is shifted with respect to the real scatterer position. This error will be investigated further in the next section. 


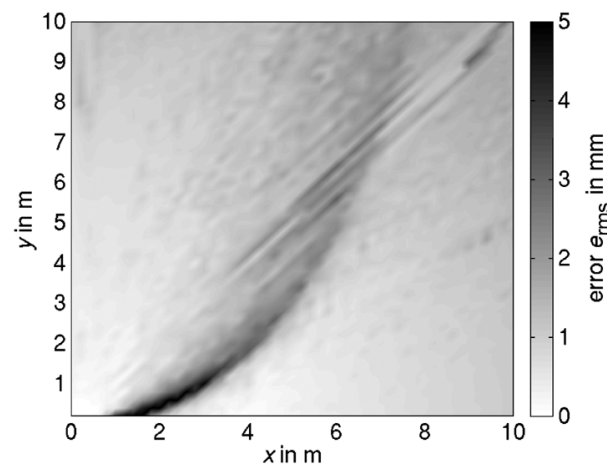

(a)

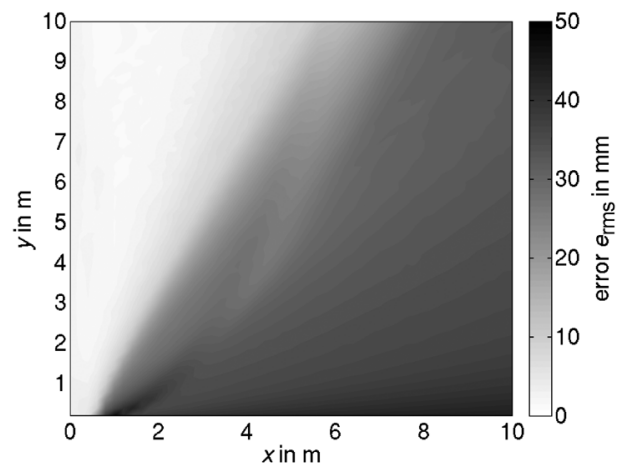

(c)

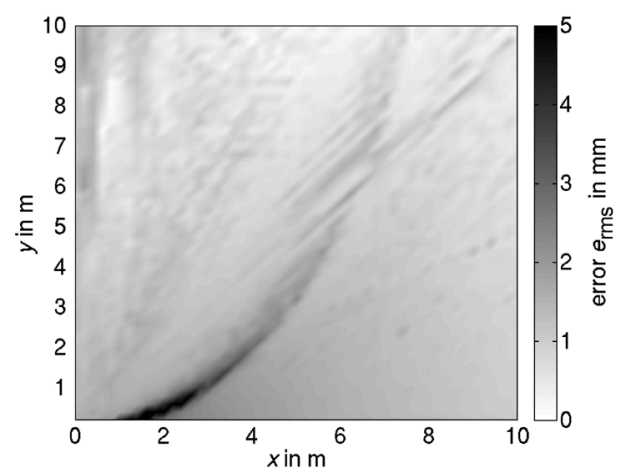

(b)

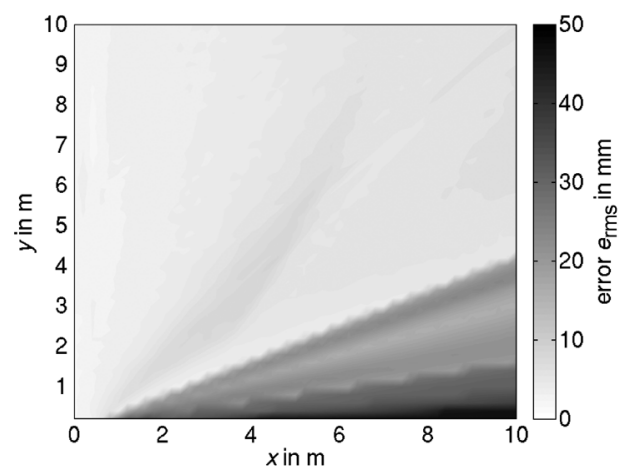

(d)

Fig. 8. Position error $e_{\text {rms }}$ versus scatterer position estimated from simulations. (a) Ideal omnidirectional antenna; (b) monocone antenna; (c) horn antenna; (d) Vivaldi antenna.

\section{B. Position Error}

For the analysis of the position error in the migrated image a series of simulations is performed. The target is placed at different positions in a square area between 0 and $10 \mathrm{~m}$ in $x$-direction and in $y$-direction. For each point, the position of the object is determined from the migrated image and compared to the original position of the target. The position error $e_{\mathrm{rms}}$ is calculated as the mean square of the deviation in $x$ - and $y$-directions. In Fig. 8 the position errors for the 4 antennas are shown. As the radiation patterns of all antennas are nearly symmetric, also the position errors for the target positions placed symmetrically to the normal of the sensor track are the same. Therefore only positive $x$-coordinates are shown.

Both, the ideal omnidirectional antenna and the monocone antenna cause no considerable errors. The observed errors are mostly smaller than $5 \mathrm{~mm}$. The horn and Vivaldi antenna cause small errors for the objects near to the normal direction of the sensor track but for the objects located at larger angles the error values increase to a few centimeters. The area with small errors is narrower for the horn antenna.

The largest error was found for the target positions at $x$-coordinates outside the sensor track. The positioning precision for these points can be improved by applying a longer sensor track so that the target points are placed within the range of the track.

To assess the influence of the spacing $d_{\mathrm{TxRx}}$ between the Tx and Rx antennas on the position error, simulations with other antenna spacings have been done for some sample points. The results for the horn antenna in Table II show that the influence of the antenna spacing is limited. The errors at positions off the
TABLE II

POSITION ERROR $e_{\mathrm{rms}}$ IN mm VERSUS ANTENNA SPACING FOR THE HORN ANTENNA

\begin{tabular}{cc|cccc}
\hline \multicolumn{2}{l|}{ Target position in $\mathrm{m}$} & \multicolumn{4}{|c}{$d_{\text {TxRx }}$ in $\mathrm{cm}$} \\
$x$ & $y$ & 5 & 15 & 45 & 135 \\
\hline 0 & 1 & 3.6 & 3.6 & 3.9 & 10.9 \\
0.71 & 0.71 & 11.0 & 10.7 & 11.2 & 19.1 \\
0.87 & 0.50 & 29.9 & 29.2 & 27.2 & 20.6 \\
\hline
\end{tabular}

normal of the sensor track are slightly smaller and the errors at positions near to the normal of the sensor track are slightly higher for wider antenna spacing, but the difference is significant only for the largest spacing. For the Vivaldi and monocone antenna this influence is even smaller.

In the case of large antennas an additional position error is possible if the radiation center of the antenna is not in the origin of the coordinate system. Therefore additional simulations have been done for the broadband horn antenna, in which the origin of the coordinate system was shifted with respect to the radiation center of the antenna. Although the errors for the targets placed near to the normal of the sensor track stay small, the errors for targets placed at larger angles grow rapidly. For a target at the position $p(x, y)=(0.71 \mathrm{~m}, 0.71 \mathrm{~m})$ the error of $e_{\mathrm{rms}}=10.7 \mathrm{~mm}$ has been observed if the antenna radiation center is placed at the origin of the coordinate system. The error increases to $e_{\mathrm{rms}}=68.6 \mathrm{~mm}$ if the radiation center is placed at the position $p(x, y)=(0 \mathrm{~m}, 0.1 \mathrm{~m})$ and to $e_{\mathrm{rms}}=86 \mathrm{~mm}$ if the radiation center is placed at the position $p(x, y)=(0 \mathrm{~m}, 0.2 \mathrm{~m})$. The errors for the target at the position $p(x, y)=(0 \mathrm{~m}, 1 \mathrm{~m})$ remain approximately the same. 


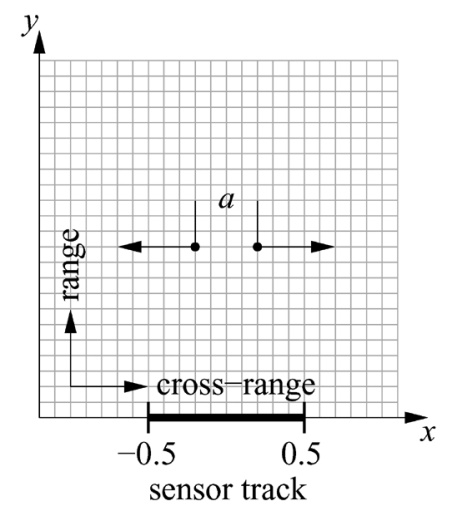

Fig. 9. Scenario for resolution estimation (cross-range).

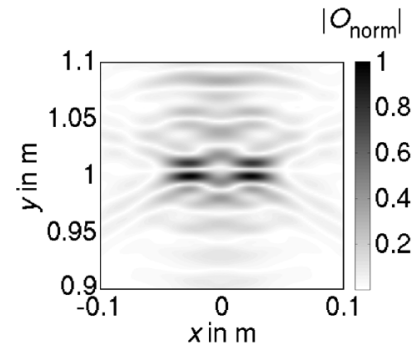

(a)

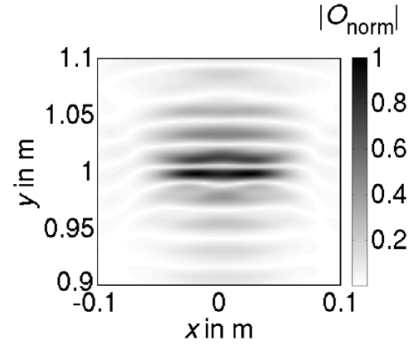

(b)
Fig. 10. Image of two targets at $p(x, y)=(0 \pm 0.025 \mathrm{~m}, 1 \mathrm{~m})$. (a) Monocone antenna; (b) horn antenna.

\section{Resolution}

For the evaluation of the available resolution two point scatterers are placed in front of the sensor (cf. Fig. 9). The multiple scattering processes between the two objects are neglected. Both, range and cross-range directions are considered. In the cross-range case the point scatterers are placed along the $x$-axis and in the range case the targets are placed along the $y$-axis at different positions in front of the sensor track. To find the resolution the distance $a$ between the scatterers is increased until the two targets can be distinguished in the image.

It is assumed that two targets are distinguishable when in the image two local maxima are separated by a local minimum, which is at least $3 \mathrm{~dB}$ below both maxima. The obtained resolution depends on the size of the single target image and is thus dependent on the antenna radiation pattern. As an example an image of two targets spaced by $5 \mathrm{~cm}$ at the distance of $1 \mathrm{~m}$ from the sensor track is shown in Fig. 10. The images have been obtained from simulations with the monocone antenna and with the horn antenna. The horn antenna results in a wide image and thus the distance between the targets has to be larger in order to distinguish them, whereas in the case of the monocone antenna the image of the target is very compact with a better resolution.

The resolution is also dependent on the position of the targets. In Fig. 11 the targets are placed at an angle of $45^{\circ}$ with respect to the middle of the sensor track. In the case of the monocone antenna both images can be distinguished already at a spacing of $2 \mathrm{~cm}$.

In Fig. 12 the cross-range and range resolution for the monocone and the horn antenna are shown for targets in the half space

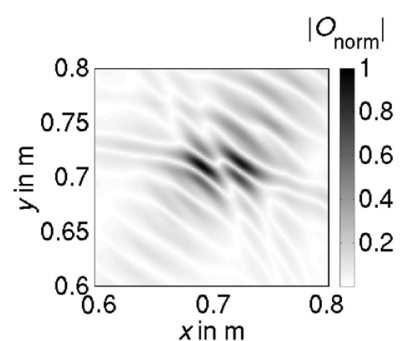

(a)

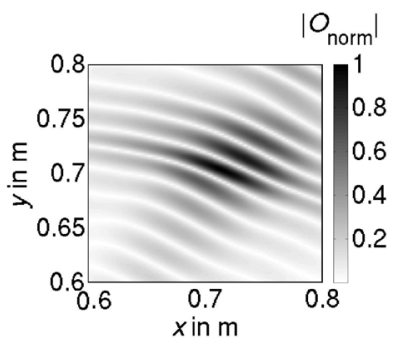

(b)
Fig. 11. Image of two targets at $p(x, y)=(0.707 \pm 0.01 \mathrm{~m}, 0.707 \mathrm{~m})$. (a) Monocone antenna; (b) horn antenna.

in front of the sensor track. The results for the omnidirectional antenna are nearly identical to the results for the monocone antenna. The performance of the Vivaldi antenna is not significantly worse than of the monocone antenna.

As the results are symmetric, only the results for positive $x$-coordinates are shown. In the cross- range case better resolutions are achievable for the targets placed at larger angles with respect to the normal of the sensor track or in the range case for the targets placed near to the normal. This is, however, caused by the used algorithm, since this effect is also visible for the ideal omnidirectional antenna. The larger the angle between the crossing arches the better resolution is possible. If the targets are placed parallel to the $x$-axis the lowest crossing angles of the arches are directly in front of the track at large distances. If the targets are placed perpendicular to the sensor track the lowest crossing angles are at the line of the sensor track. Nevertheless, slightly lower resolution can be observed for the horn antenna at all target positions. Such an antenna limits the number of positions on the sensor track, at which the scatterer contributes to the received signal. The image of a target becomes broader and thus the distance, at which two targets can be resolved becomes larger.

The achieved resolutions in range and cross-range can be additionally influenced by system parameters like bandwidth or track length. The influence of the Tx-Rx antenna spacing on the resolution is limited. The bandwidth of the imaging system influences directly the range resolution. A larger bandwidth enables narrower pulses in time domain, which yields more precise time estimation. In the migration algorithm the hyperbolic contributions from each sensor positions are also narrower and the crossing point between the arches is thus better defined. For example if the ECC band $(6 \mathrm{GHz}-8.5 \mathrm{GHz})$ is used the resulting range resolution is approximately three times worse for all considered antennas.

In case of omnidirectional antennas the cross-range resolution can be improved by using larger synthetic aperture. For the omnidirectional antenna as well as for the monocone antenna the cross-range resolution $\Delta x$ at the position $p(x, y)=$ $(0 \mathrm{~m}, 1 \mathrm{~m})$ is approximately $4 \mathrm{~cm}$ for a $1 \mathrm{~m}$ long track, $\Delta x=$ $2.5 \mathrm{~cm}$ for a $2 \mathrm{~m}$ long track and $\Delta x=1.9 \mathrm{~cm}$ for a $4 \mathrm{~m}$ long track. If a directive antenna is used (e.g. horn antenna), the resolution does not change significantly with the track length, as the contributions from targets seen at a high angle by the sensor antenna are strongly attenuated. 


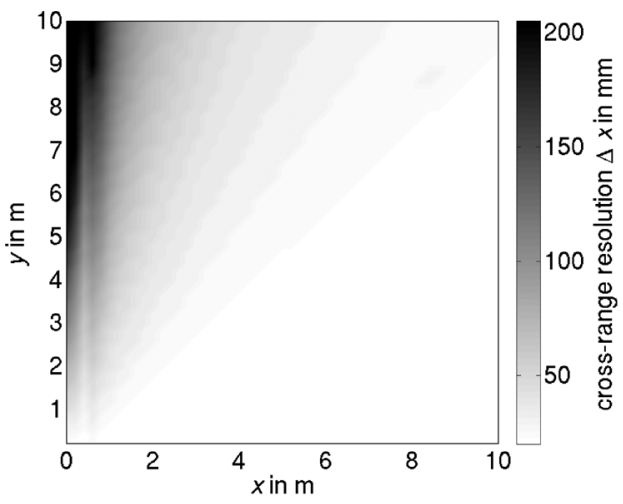

(a)

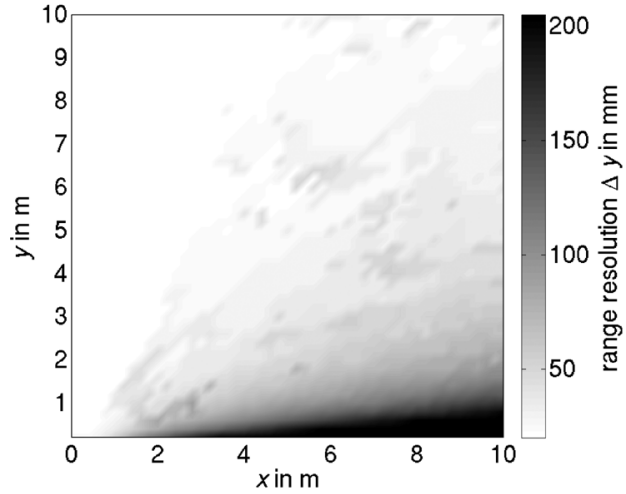

(c)

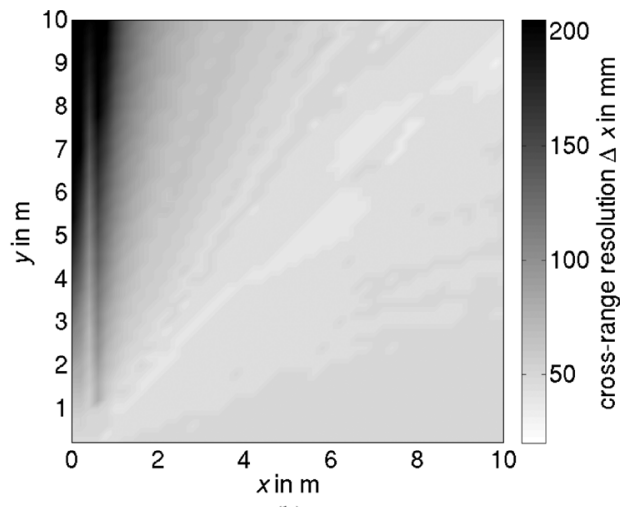

(b)

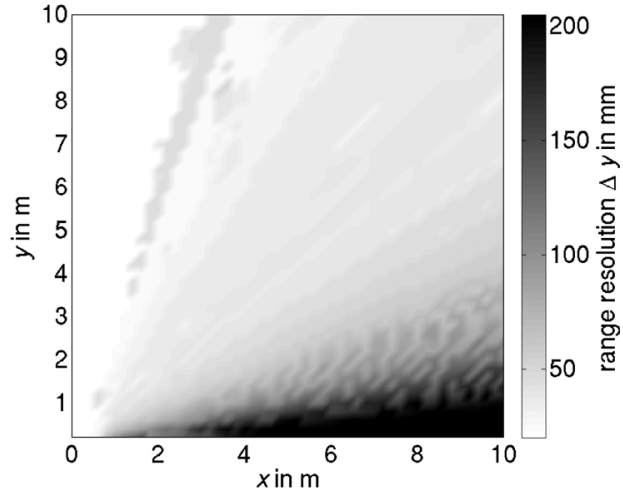

(d)

Fig. 12. Resolution versus scatterer position estimated from simulations. (a) Monocone-cross-range; (b) horn-cross-range; (c) monocone-range; (d) horn-range.

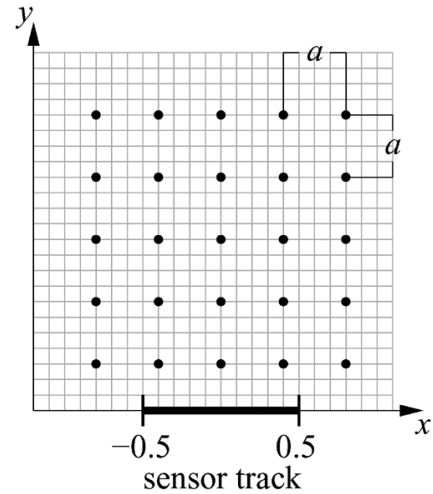

Fig. 13. Scenario for coverage estimation.

\section{Coverage}

In the last step the coverage is examined using identical point scatterers placed on a grid with spacing of $a=0.5 \mathrm{~m}$. In the $x$-direction the scatterers are placed also outside of the sensor track (cf. Fig. 13). Two scenarios are considered: in the first scenario a near scatterer is present in the second scenario a single, distant scatterer is considered. No additional measures for range or antenna pattern compensation are taken.

If a near scatterer is present in the scenario it generates strong artifacts in the migrated image. The amplitude of such artifacts, relative to the amplitude of the target image is inversely proportional to the number of sensor positions. The amplitude of other targets has to be higher than the amplitude of the artifacts if they are to be detected. Thus the image artifacts limit the possible dynamic range of the system. In the case of the considered system with 51 sensor positions this dynamic range is approximately

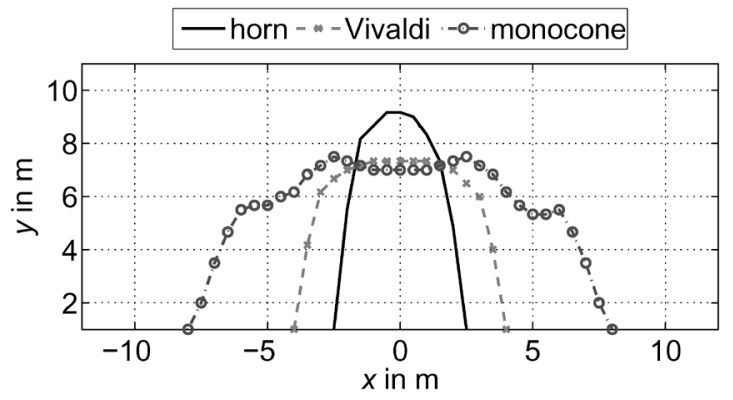

Fig. 14. Coverage areas for different antennas estimated from simulations.

$34 \mathrm{~dB}$. Thus the coverage depends on the number of sensor positions and on the distance to the nearest target.

In the following the nearest scatterer is placed $1 \mathrm{~m}$ to the sensor track at the position $p(x, y)=(0 \mathrm{~m}, 1 \mathrm{~m})$. Fig. 14 shows the resulting coverage areas for the monocone, Vivaldi and horn antenna. The coverage areas for the ideal isotropic antenna is identical with the coverage for the monocone antenna. There is a strong correspondence to the pattern of the antenna: The omnidirectional antennas are able to localize also the targets that are far from the track in cross-range, whereas the cross-range coverage of directive antennas is strongly limited.

The improvement of the range coverage for a directive antenna is limited in this case because also the artifacts of the strongest contribution are amplified. The small improvement is due to the high attenuation of directive antennas at larger angles. The nearest scatterer is visible for the sensor at low angles only on a short part of the track. For sensor positions at the edges of the track the contribution of the nearest scatterer is strongly 


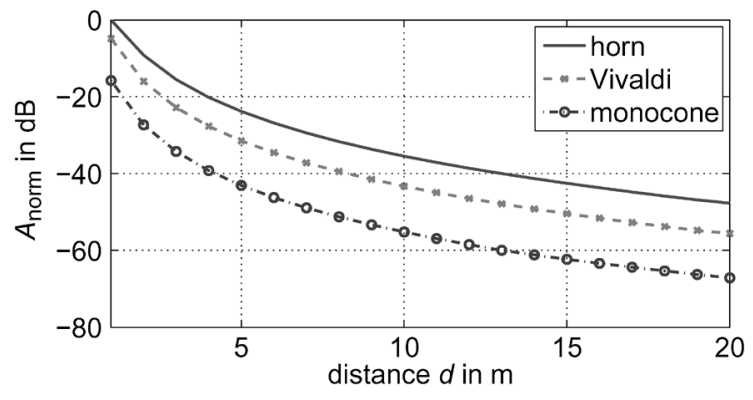

Fig. 15. Normalized amplitude of the reference target.

attenuated. On the other hand, the scatterer, which is placed further away is seen at lower angles and its contributions are less attenuated by the antenna.

If only one distant scatterer is present in the considered area the gain of a directive antenna may improve the signal to noise ratio and help to detect the scattered signal. To compare the performance of the antennas, single targets placed at positions $p(x, y)=(0 \mathrm{~m}, d), d=1 \mathrm{~m}-20 \mathrm{~m}$ have been simulated and the target amplitudes $A$ have been extracted from the images. Fig. 15 shows these amplitudes for three considered real antennas. The values are normalized with respect to the target amplitude for the horn antenna at the distance of $1 \mathrm{~m}$.

For the distant scatterers the horn antenna delivers a target image, which is nearly $20 \mathrm{~dB}$ stronger than the image with the monocone antenna and about $8 \mathrm{~dB}$ stronger than the image delivered by the Vivaldi antenna. Thus in case of a directive antenna like the horn antenna the range coverage of the imaging is increased. The exact values of the coverage in the case of a single distant scatterer are also dependent on the radar cross section of the target, the sensitivity of the receiver and the noise level.

\section{CONCLUSION}

The influence of the antenna on the shape of the target image, position error, resolution and coverage of an ideal imaging system is analyzed in this work. It is shown that the target image shape and its position in the migrated image are strongly dependent on the angular variance of the transient response of the antenna. Strongly directive antennas deliver broader target images and cause large position errors for the scatterers placed off the normal of the sensor track. This effect is even stronger if the radiation center of the antennas is shifted with respect to the origin of the coordinate system used for imaging.

The resolution is influenced mostly by antenna-independent system parameters like bandwidth, track length, or the distance of the target to the sensor track, however, directive antennas deliver slightly lower resolution in range and cross-range.

Omnidirectional antennas generally yield a larger coverage in the cross-range than directive antennas. The most severe coverage limitation is caused by the scatterers in the proximity of the sensor track. The artifacts from the images of the near targets limit the available dynamic range of the image. On the other hand if only far scatterers are present the higher gain antennas offer considerably better coverage in range than the omnidirectional ones.

\section{REFERENCES}

[1] Revision of Part 15 of the Commission's Rules Regarding Ultra Wideband-First Report and Order 02-48 Federal Communications Commission (FCC), Apr. 2002.

[2] Decision 2007/131/EC on Allowing the Use of the Radio Spectrum for Equipment Using Ultra-Wideband Technology in a Harmonised Manner in the Community European Commission, Feb. 2007.

[3] S. Gezici, Z. Tian, G. B. Giannakis, H. Kobayashi, A. F. Molisch, H. V. Poor, and Z. Sahinoglu, "Localization via ultra-wideband radios: A look at positioning aspects for future sensor networks," IEEE Signal Process. Mag., vol. 22, no. 4, pp. 70-84, Jul. 2005.

[4] R. J. Fontana, "Recent system applications of short-pulse ultra-wideband (UWB) technology," IEEE Trans. Microw. Theory Tech., vol. 52, no. 9, pp. 2087-2104, Sep. 2004.

[5] D. J. Daniels, Ground Penetrating Radar, 2nd ed. London. U.K.: Inst. Elect. Eng., 2006.

[6] S. Hantscher, B. Praher, A. Reisenzahn, and C. G. Diskus, "Comparison of UWB target identification algorithms for through-wall imaging applications," in Proc. 3rd Eur. Radar Conf., Manchester, GB, Sep. 2006, pp. 104-107.

[7] S. Kidera, T. Sakamoto, and T. Sato, "A high-resolution 3-D imaging algorithm with linear array antennas for UWB pulse radar systems," presented at the IEEE Antennas and Propag. Society Int. Symp., Albuquerque, NM, Jul. 2006.

[8] R. Zetik, J. Sachs, and R. Thoma, "Modified cross-correlation back projection for UWB imaging: Numerical examples," presented at the IEEE Int. Conf. Ultrawideband ICU, Zurich, Switzerland, Sep. 2005.

[9] R. Zetik, J. Sachs, and R. Thomä, "Imaging of propagation environment by UWB channel sounding," presented at the XXVIIIth General Assembly of URSI, New Delhi, India, Oct. 2005.

[10] M. Porebska, J. Timmermann, C. Sturm, T. Zwick, and W. Wiesbeck, "Impact of non-ideal antennas on the performance of UWB imaging systems," presented at the Proc. 17th Int. Conf. on Microw. Radar and Wireless Commun., Wroclaw, Poland, May 2008.

[11] N. Geng and W. Wiesbeck, Planungsmethoden fuer die Mobilkommunikation (in German). Berlin; Heidelberg: Springer, 1998.

[12] A. Ishimaru, Electromagnetic Wave Propagation, Radiation and Scattering. Englewood Cliffs, NJ: Prentice-Hall, 1991.

[13] M. Porebska, C. Sturm, J. Timmermann, T. Zwick, and W. Wiesbeck, "Influence of multipath propagation on UWB imagery," presented at the IEEE Int. Conf. on Ultra-Wideband, Hannover, Germany, Sep. 2008.

[14] W. Sörgel and W. Wiesbeck, "Influence of the antennas on the ultra wideband transmission," in Proc. EURASIP J. Appl. Signal Proc., Special Issue UWB-State of the Art, Mar. 2005, pp. 296-305.

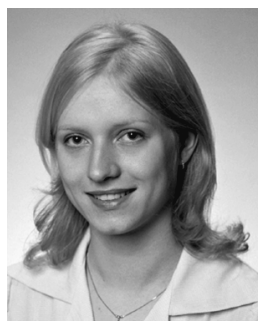

Malgorzata Janson (S'06) received the Dipl.-Ing. (M.S.E.E.) degree in electrical engineering from the Universität Karlsruhe (TH), Germany, in 2005 and the master's degree in electrical engineering from the Gdansk University of Technology, Poland, in 2005.

From 2003 to 2005, she took part in the integrated academic program Gdansk-Karlsruhe. She is currently working toward the $\mathrm{Ph} . \mathrm{D}$. degree at the Institut für Hochfrequenztechnik und Elektronik, Universität Karlsruhe (TH). Her research interests include wave propagation, channel measurement techniques, deterministic and stochastic channel modeling, and ultrawideband imaging systems. 


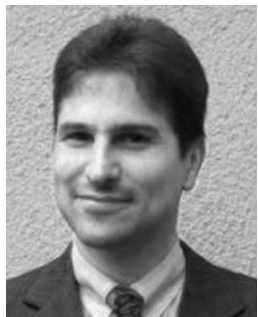

Thomas Zwick (S'95-M'00-SM'06) received the Dipl.-Ing. (M.S.E.E.) and the Dr.-Ing. (Ph.D.E.E.) degrees from the Universität Karlsruhe $(\mathrm{TH})$, Germany, in 1994 and 1999, respectively.

From 1994 to 2001, he was a Research Assistant at the Institut für Höchstfrequenztechnik und Elektronik (IHE) at the Universität Karlsruhe (TH), Germany. February 2001, he joined IBM as a Research Staff Member at the IBM T. J. Watson Research Center, Yorktown Heights, NY. From October 2004 to September 2007, he was with Siemens AG, Lindau, Germany. During this period he managed the RF development team for automotive radars. In October, he became appointed a Full Professor at the Universität Karlsruhe (TH), Germany. He is Director of the Institut für Hochfrequenztechnik und Elektronik (IHE) at the Universität Karlsruhe (TH), Germany. His research topics include wave propagation, stochastic channel modeling, channel measurement techniques, microwave material measurements, microwave techniques, millimeter wave antenna design, ultrawideband systems, wireless communication and radar system design. $\mathrm{He}$ participated as an expert in the European COST231 Evolution of Land Mobile Radio (Including Personal) Communications and COST259 Wireless Flexible Personalized Communications.

Prof. Zwick received the best paper award at the International Symposium on Spread Spectrum Techniques and Applications ISSSTA 1998. In 2005 he received the Lewis Award for the outstanding paper at the IEEE International Solid State Circuits Conference. Since 2008, he has been President of the Institute for Microwaves and Antennas (IMA). For the Carl Cranz Series for Scientific Education he served as a lecturer for wave propagation.

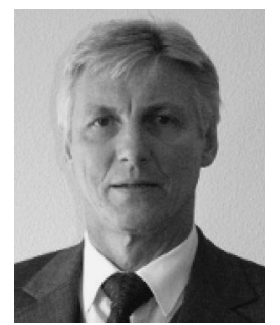

Werner Wiesbeck (SM'87-F'94) received the Dipl.-Ing. (M.S.E.E.) and Dr.-Ing. (Ph.D.E.E.) degrees from the Technical University Munich, Germany, in 1969 and 1972, respectively.

From 1972 to 1983, he was with AEG-Telefunken in various positions including that of $\mathrm{Head}$ of R\&D of the Microwave Division in Flensburg and Marketing Director Receiver and Direction Finder Division, Ulm. During this period he had product responsibility for $\mathrm{mm}$-wave radars, receivers, direction finders and electronic warfare systems. From 1983 to 2007, he was Director of the Institut für Höchstfrequenztechnik und Elektronik (IHE) at the University of Karlsruhe (TH). In 1989 and 1994, respectively, he spent a six months sabbatical at the Jet Propulsion Laboratory, Pasadena. Research topics included radar, remote sensing, wireless communication, ultrawideband and antennas. Since 2007, he has been Excellent Retired Scientist at the Karlsruhe Institute of Technology concentrating on radar, ultrawideband and mobile to mobile communications.

Dr. Wiesbeck is the recipient of a number of awards, most recently, the IEEE Millennium Award, the IEEE GRS Distinguished Achievement Award, the Honorary Doctorate (Dr. h.c.) from the University Budapest/Hungary, the Honorary Doctorate (Dr.-Ing. E.h.) from the University Duisburg/Germany and the IEEE Electromagnetics Award 2008. He is a Fellow of IEEE, an Honorary Life Member of IEEE GRS-S, a Member of the Heidelberger Academy of Sciences and a Member of Acatech (German Academy of Engineering and Technology). For the Carl Cranz Series for Scientific Education he serves as a permanent lecturer for radar systems engineering, wave propagation and mobile communication network planning. He is a member of an Advisory Committee of the EU-Joint Research Centre (Ispra/Italy), and he is an advisor to the German Research Council (DFG), to the Federal German Ministry for Research (BMBF) and to industry in Germany. He is a member of the IEEE GRS-S AdCom (1992-2000), Chairman of the GRS-S Awards Committee (1994-1998, 2002-), Executive Vice President IEEE GRS-S (1998-1999), President IEEE GRS-S (2000-2001), Associate Editor IEEE-AP Transactions (1996-1999), past and present Treasurer of the IEEE German Section (1987-1996, 2003-2007). He has been General Chairman of the 1988 Heinrich Hertz Centennial Symposium, the 1993 Conference on Microwaves and Optics (MIOP'93), the Technical Chairman of International $\mathrm{mm}$-Wave and Infrared Conference 2004, Chairman of the German Microwave Conference GeMIC 2006 and he has been a member of the scientific committees and TPCs of many conferences. 